\title{
Fünf Thesen für eine transformative Anpassungspolitik
}

\author{
Politik zu Klimawandel und Anpassung ist \\ geprägt von widerstreitenden Interessen und \\ Machtverhältnissen. Sie unterliegt der Gefahr, \\ bestehende Ungleichheiten zu verschärfen oder \\ neue zu schaffen. Eine sozial gerechte An- \\ passungspolitik erfordert eine umfassende \\ gesellschaftliche Transformation - fünf Thesen. \\ Von Achim Brunnengräber und Kristina Dietz
}

Sänds ich anpassen bedeutet sprichwörtlich Verhaltensweisen zu ändern, um angemessen auf gegebene Umstände und Rahmenbedingungen reagieren zu können. Anpassung als Begriff verweist also weniger auf progressive Handlungsschritte. In ihm klingt vielmehr ein Strukturkonservativismus an, wenn vor allem Reformen von etablierten Institutionen im Vordergrund stehen und nicht deren grundsätzliche Neugestaltung. Es sind jedoch gerade bestehende soziale, politische und ökonomische Prozesse und Strukturen, die für den Klimawandel und die daraus entstehende Krise ursächlich sind und für viele Menschen dramatische Folgen haben. Wenn von Anpassung die Rede ist, muss deshalb gefragt werden, wer sich überhaupt an was anpassen soll. Eine Beantwortung dieser Frage muss die gesellschaftlichen Verhältnisse insgesamt in den Blick nehmen.

Gegenüber einer strukturkonservativen Perspektive ist dann eine grundsätzlich veränderte normative Orientierung und erweiterte Perspektive erforderlich. Nicht nur die Überwindung der Krise für Menschen oder die Betroffenen im Allgemeinen ist dann entscheidend, sondern gerade auch die Bedeutung von Institutionen und Strukturen, die einen gerechten Umgang mit den Folgen des Klimawandels verhindern. Dann geraten Einkommensstrukturen, Interessen, Machtverhältnisse und politische Teilhabemöglichkeiten in den Blick der Anpassungspolitik. Die Herausforderung besteht insbesondere darin, die Interessen zwischen Anpassung und dem Verbrauch fossiler Energien zu identifizieren sowie die strukturellen Hindernisse zu berücksichtigen, die einer Reduzierung von globalen Armuts- und Ungleichheitsverhältnissen entgegenstehen. Beides sind Vorbedingungen für erfolgreiche Anpassungsmaßnahmen.

Vor diesem Hintergrund argumentieren wir im vorliegenden Beitrag, dass sozial gerechte Anpassungspolitik nur dann erzielt werden kann, wenn diese in ein umfassendes Projekt der gesellschaftlichen Transformation eingebettet ist (1). Klimawandel und Anpassung müssen dafür, jenseits ihrer stofflich-mate- riellen Bedingungen, als politische Begriffe und Prozesse verstanden werden. Denn bereits ihre Definition ist umkämpft und von Interessen geprägt. Politische Maßnahmen, die ausgehend von bestimmten Problemdeutungen getroffen werden, wirken außerdem gesellschaftlich sehr unterschiedlich. Mitunter bergen sie die Gefahr, Ungleichheiten zu verschärfen oder neue zu schaffen. Auch vermeintlich objektives Wissen über Klimawandelfolgen und über die richtigen Maßnahmen zur Anpassung muss immer wieder kritisch hinterfragt werden (Grothmann et al. 2011). Neben der Suche nach Anpassungsmaßnahmen im Umgang mit den Folgen des Klimawandels muss es schließlich um die Suche nach einer veränderten normativen Handlungsorientierung gehen, die die soziale Frage wieder stärker ins Zentrum der ökologischen Krise rückt. Um dies zu verdeutlichen, stellen wir fünf Thesen zur Diskussion.

\section{Vielfalt der Wandelprozesse berücksichtigen}

Eine anerkannte Definition von Anpassung findet sich im dritten Sachstandsbericht des zwischenstaatlichen Ausschusses für Klimaänderungen (IPCC). Anpassung wird hier sinngemäß verstanden als die Veränderung sozialer, ökologischer und ökonomischer Systeme in Reaktion auf „aktuelle und erwartete Klimaveränderungen“ (Smit/Pilifosova 2001). Mit dieser Fokussierung wird der Blick auf die gegenwärtigen und zukünftigen Klimawandelfolgen gerichtet. Für ihre Bearbeitung schlägt der IPCC top down-orientierte technische und infrastrukturelle Anpassungsmaßnahmen vor, die ein breites Feld betreffen: vom Naturschutz, über Wassermanagement bis Mobilität, Bauen oder Wohnen. Ziel ist es, Schäden für Mensch und Natur abzumildern und Folgen beherrschbar zu machen. Ein derartiges Verständnis von Anpassung abstrahiert von nicht klimatischen Wandelprozessen sowie gesellschaftlichen und politischen Strukturen. Diese bestimmen jedoch gerade, ob der Umgang mit den Folgen des Klimawandels gesellschaftlich überhaupt möglich ist und sozial gerecht erfolgen kann.

\section{Kontextualisierungen vornehmen}

Zwar ist es durchaus sinnvoll, wie in den meisten Anpassungsstrategien auch vorgesehen, Wissenslücken über Klimawandelfolgen oder Anpassungserfordernisse zu schließen. Damit wird aber der vielschichtige soziale Kontext nicht ausreichend berücksichtigt, in dem Maßnahmen umgesetzt werden. Hilfreich ist hier das Konzept der Verwundbarkeit. Die Klimarahmenkonvention versteht Verwundbarkeit, die quasi der $\Rightarrow$ 


\section{„Einer transformativen \\ Anpassungspolitik stehen machtvolle Interessen gegenüber, die in der Anpassung eine Absicherung bestehender gesellschaftlicher Verhältnisse sehen."}

Anpassungsnotwendigkeit voraus geht und sie bedingt, primär als outcome, also Ergebnis des Klimawandels und vernachlässigt somit soziale Vermittlungsmechanismen. Vulnerabilitätsanalysen müssen deshalb nicht nur einen erweiterten Kontext berücksichtigen, sondern überhaupt erst die Vielschichtigkeit der Anpassungserfordernisse in Erfahrung bringen. Daraus leiten sich die sozialen, politischen oder sozio-ökonomischen sowie die institutionellen Aufgaben ab, mit denen sich Anpassungspolitik konfrontiert sieht (Dietz 2011). Karen O'Brien hat dafür das Konzept der kontextualisierten Vulnerabilität entwickelt (O'Brien et al. 2007).

\section{Das Politische stärker fokussieren}

Anpassung muss als ein politischer Prozess verstanden werden. Vier Aspekte gilt es in diesem Zusammenhang hervorzuheben. Erstens ist es nicht nur wichtig zu verstehen, wer welche Maßnahmen aus welchen Gründen ergreift, oder zu identifizieren, welche technischen Maßnahmen Schäden abmildern. Wichtig ist auch zu verstehen, wer überhaupt in der Lage ist, an einem Planungs- und Entscheidungsprozess teilzunehmen beziehungsweise Anpassungsmaßnahmen zu definieren und umzusetzen. So kann beispielsweise nicht auf mögliche Folgen des Klimawandels vorbeugend reagiert werden, wenn Anpassungskapazitäten fehlen oder politische Repräsentations- und Artikulationsräume dafür nicht gegeben sind.

Zweitens sind formale, vom politisch-administrativen System ausgehandelte Maßnahmen grundsätzlich zu unterscheiden von Formen der Anpassung, die unabhängig von diesen individuell oder kollektiv auf lokal-regionaler Ebene stattfinden. Beispiele hierfür sind veränderte landwirtschaftliche Anbauweisen in der Subsistenzwirtschaft, temporäre oder permanente Migration oder die Umstrukturierung der Einkommens- und Lebensverhältnisse häufig unter Inkaufnahme von neuen Abhängigkeitsverhältnissen. Dazu gehört insbesondere die in Form von Nahrungsmitteln ausgezahlte Landarbeit (Dietz 2011, anhand der Ausführungen zu Tansania). Governance-Maßnahmen, die gesteuerte Anpassungspolitiken in den Vordergrund stellen, berücksichtigen diese gesellschaftlichen Zusammenhänge auf lokaler und individueller Ebene kaum.
Drittens beeinflussen Fragen der gesellschaftlichen Verteilung von Gestaltungs- und Entscheidungsmacht die Anpassungskapazität. Einzelne empirische Arbeiten belegen, dass Anpassung ein primär politischer und konfliktiver Prozess der Aushandlung um die Nutzung von und den Zugang zu Ressourcen zu Land oder zu Wasser (Eriksen/Lind 2008) ist. Politische Dimensionen werden auch dann sichtbar, wenn eine mangelnde politische Einflussnahme sozialer Akteure das Risiko erhöht, von Anpassungspolitiken negativ betroffen zu sein oder in Krisensituationen staatliche Unterstützungsmaßnahmen nicht aktivieren zu können.

Zugleich können, viertens, Maßnahmen, die als Anpassung an den Klimawandel deklariert werden, legitimatorische Funktionen übernehmen, indem sie Maßnahmen, die ansonsten schwer durchsetzbar sind, größere Akzeptanz verschaffen.

Umso wichtiger ist es, im Sinne einer partizipativen Anpassung, die Belange der betroffenen Bevölkerung mit einzubeziehen. Anpassung droht zu einem top down-Prozess reduziert zu werden, wenn die Beteiligung nur in Form von einmaligen Konsultationen erfolgt. Ein hierüber hinausreichendes Verständnis von Partizipation versteht Anpassung als alltägliche Herausforderung und Praxis. Zu fragen ist, welche Belange lokale Bevölkerungsgruppen selbst artikulieren, welche Anpassungsmaßnahmen bereits stattfinden und welche Unterstützung gewollt ist. Anpassung wäre dann ein Prozess der Ermächtigung und Partizipation, des empowerment, oder der Verringerung von Armut, bei denen nicht nur technisch-methodische Planungsprozesse zum Tragen kommen.

\section{Kohärente Governance-Strukturen schaffen}

Anpassungspolitik sieht sich mit einer Unübersichtlichkeit an Institutionen, einer nichtkohärenten Infrastruktur, mangelnden finanziellen Mitteln und vielfältigen Konzepten konfrontiert, sprich mit Governance-Defiziten. So hat sich in der internationalen Klimapolitik mittlerweile ein dichtes Geflecht an Gremien, Regeln und Finanzierungsfonds gebildet, das an Komplexität kaum noch zu überbieten ist. In der Vielfalt der Governance-Strukturen auf regionaler, nationaler und internationaler Ebene spiegelt sich quasi die Breite der Definition von Anpassung wider. Organisationen wie die Weltbank, der Internationale Währungsfonds und die Organisation für wirtschaftliche Zusammenarbeit und Entwicklung oder das Umweltprogramm der Vereinten Nationen entwickeln jeweils eigene Anpassungsstrategien, die mit dem „Klimasystem“ der Vereinten Nationen nicht unbedingt kohärent sind. Ähnliches gilt für die Klimafinanzierung. Über 25 bi- und multilaterale Fonds wurden geschaffen, wobei sich in der Entwicklungspolitik eine zentrale Frage darum dreht, ob diese Mittel zusätzlich zu den offiziellen Hilfsgeldern gezahlt werden oder nur eine Umschichtung der Entwicklungsgelder darstellen (2).

Auch Regierungen und privatwirtschaftliche Akteure sind Teil der unübersichtlichen Gemengelage, in der es um die Verteilung des finanziellen Kuchens, konkurrierende und auch sich 
widersprechende Anpassungsmaßnahmen sowie um die Frage nach dem eigentlichen Adressatenkreis dieser Maßnahmen geht. Sowohl in den Klimaverhandlungen als auch außerhalb davon ist Anpassungspolitik in hohem Maße von polit-ökonomischen Interessen geprägt. Und es wird ausdrücklich vor dem Risiko einer weiter "steigenden Fragmentierung“ gewarnt (SEF 2011). Eine kohärentere Governance-Struktur scheint nur möglich und sinnvoll, wenn sie, wie oben gefordert, mit der Herausbildung einer neuen normativen Orientierung einhergeht.

\section{Klimagerechtigkeit verwirklichen}

Die gesellschaftlichen Widersprüche, die in die internationale Klimapolitik eingeschrieben sind und zu denen sozioökonomische Ungleichheiten, Luxus- und Armutsemissionen oder ungleiche Chancen zur Anpassung gehören, werden erst allmählich im Klimadiskurs berücksichtigt. Zunächst wurde auch hier global gedacht. Gemäß der Klimarahmenkonvention von 1994 soll die internationale Staatengemeinschaft Maßnahmen zum Klimaschutz „auf der Grundlage der Gerechtigkeit und entsprechend ihren gemeinsamen, aber unterschiedlichen Verantwortlichkeiten und ihren jeweiligen Fähigkeiten“ (Art. 3.1) ergreifen. Doch das Protokoll ist blind gegenüber Gerechtigkeitsfragen, die über die einfache Unterscheidung zwischen Industrie- und Entwicklungsländern hinausreichen. Deshalb wurde das zwischenstaatliche Gerechtigkeitsprinzip erweitert. Klimapolitik wird dann als gerecht verstanden, wenn sich die Pro-Kopf-Emissionen auf möglichst geringem Niveau angleichen.

Industrieländer müssten, um dieses Ziel zu erreichen, die Emissionen pro Kopf drastisch senken, die Entwicklungsländer dürften moderat zulegen. Auch wenn in diesem erweiterten Verständnis Grundsätze liberaler Gerechtigkeitsprinzipien erkennbar sind, greift auch dieses Verständnis noch zu kurz. Denn mit der Forderung nach einer individuell gleichen Lasten- sowie Nutzenverteilung des Gemeinschaftsgutes Atmosphäre wird einem Denkmuster gefolgt, in dem Ansätze der Nichtverschmutzung und der nicht marktförmigen Bearbeitung der Klimakrise unberücksichtigt bleiben. Gesellschaftliche Entwicklung in Entwicklungs- wie Industrieländern kann folglich mit der effizienteren Nutzung fossiler Energieträger, mit globalem Standortwettbewerb und weltwirtschaftlichem Wachstum weitergehen (Brunnengräber/Dietz 2012). Den anspruchsvollen Gerechtigkeitsansatz weitergedacht, müsste eine umfassende gesellschaftliche Transformation auch die Maßstäbe der Moderne von Wachstum, Ressourcenverbrauch und Wohlstand neu verhandeln.

\section{Gesellschaftliche Transformationen anregen}

Klimagerechtigkeit, die Energiewende, der Ausbau Erneuerbarer Energien, die Verringerung der globalen Armut und die politische Teilhabe werden somit zu Leitlinien einer Anpassungspolitik, die gesellschaftlichen Wandel und Anpassungsmaßnahmen zu verknüpfen versucht. Wir fordern deshalb die

Entwicklung einer transformativen Anpassungspolitik. Dem stehen allerdings machtvolle Interessen gegenüber, die in der Umsetzung von Anpassungspolitiken eher eine Absicherung bestehender gesellschaftlicher Verhältnisse sehen. Daraus leiten sich die Herausforderungen an eine sensitive, partizipative und kontextualisierte Anpassungspolitik ab, die in diesem Beitrag skizziert wurden. Ohne eine veränderte normative Orientierung wird Anpassung dagegen kaum das Paradies der Beliebigkeit verlassen können und auch weiterhin ein breites Handlungsfeld für die unterschiedlichsten Interessen bieten.

\section{Anmerkungen}

(1) Erste Gedanken zu diesem Beitrag wurden von Achim Brunnengräber beim 5. Workshop zur sozialwissenschaftlichen Klimaanpassungsforschung im November 2011 im Leibniz-Institut für ökologische Raumentwicklung e. V. (IÖR) in Dresden vorgetragen. Wir danken den Workshopteilnehmerlnnen für ihre kritischen Rückmeldungen. Außerdem danken wir Philip Bedall für hilfreiche Kommentare.

(2) Für detaillierte Informationen siehe im Internet: http://www.climatefundsupdate.org

\section{Literatur}

Brunnengräber, A./ Dietz, K.: Klimagerechtigkeit. In: Brand, U. et al. (Hrsg.): $A B C$ der Alternativen 2.0. Von Alltagskultur bis Zivilgesellschaft. Hamburg 2012, S. 140-141.

Dietz, K.: Der Klimawandel als Demokratiefrage. Sozial-ökologische und politische Dimensionen von Vulnerabilität in Nicaragua und Tansania. Münster 2011.

Eriksen, S./ Lind, J.: Adaptation as a Political Process: Adjusting to Drought and Conflict in Kenya's Drylands. In: Environmental Management 2008. o.S. Internet: http://www.springerlink.com/content/76111 k554l742105/

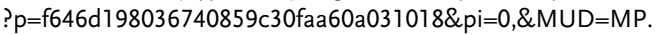

Grothmann, T./ Daschkeit, A./ Felgentreff, C./ Görg, Ch./ Horstmann, B./ Scholz, I./ Tekken, V. (2011): Anpassung an den Klimawandel - Potenziale sozialwissenschaftlicher Forschung in Deutschland. In: GAIA 20, 2/2011, S. 84-90.

O'Brien, K./ Eriksen, S./ Nygaard, L./ Schjolden, A.: Why different interpretations of vulnerability matter in climate change discourses. In: Climate Policy 7, 1/2007, S. 73-88.

Smit, B./ Pilifosova, O.: Adaptation to Climate Change in the Context of Sustainable Development and Equity. In: McCarthy, J. J. et al. (Hrsg.): IPCC Third Assessment Report: Climate Change 2001: Working Group II: Impacts, Adaptation and Vulnerability. Cambridge 2001, S. 879-912.

IAUTOREN + KONTAKT

Dr. Achim Brunnengräber ist Privatdozent am Fachbereich Politik- und Sozialwissenschaften der Freien Universität Berlin.

E-Mail: Achim.Brunnengraeber@FU-Berlin.de

Dr. Kristina Dietz ist wissenschaftliche Mitarbeiterin am Lateinamerika-Institut der Freien Universität Berlin.

E-Mail: Kristina.Dietz@FU-Berlin.de
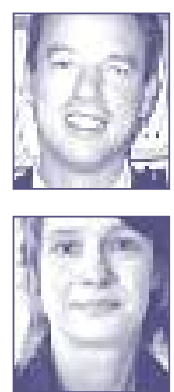
Copyright (C) 2012, IÖW und oekom Verlag. Die Nutzung des Artikels ist Abonnenten von Ökologisches Wirtschaften vorbehalten. Nachdruck und Vervielfältigung des Artikels einschließlich Speicherung und Nutzung auf optischen und elektronischen Datenträgern nur mit Zustimmung der Redaktion von Ökologisches Wirtschaften (http://www.oekologischeswirtschaften.de). 